\title{
Neutrophil extracellular trap formation and nuclease activity in septic patients
}

\author{
Linda E. Cox ${ }^{1 *} \mathbb{D}$, Kai Walstein ${ }^{1}$, Lena Völlger², Friederike Reuner ${ }^{2}$, Alexandra Bick', Annika Dötsch', Andrea Engler',
} Jürgen Peters', Maren von Köckritz-Blickwede ${ }^{2,3}$ and Simon T. Schäfer ${ }^{1,4}$

\begin{abstract}
Background: There is little knowledge, whether in patients with sepsis neutrophil extracellular trap (NET) formation and NET degrading nuclease activity are altered. Thus, we tested the hypotheses that 1) NET formation from neutrophils of septic patients is increased compared to healthy volunteers, both without stimulation and following incubation with mitochondrial DNA (mtDNA), a damage-associated molecular pattern, or phorbol 12-myristate 13-acetate (PMA; positive control) and 2) that serum nuclease activities are increased as well.
\end{abstract}

Methods: Following ethic committee approval, we included 18 septic patients and 27 volunteers in this prospective observational trial. Blood was withdrawn and NET formation from neutrophils was analyzed in vitro without stimulation and following incubation with mtDNA (10 $\mathrm{mg} /$ well) or PMA ( $25 \mathrm{nmol})$. Furthermore, serum nuclease activity was assessed using gel electrophoresis.

Results: In contrast to our hypothesis, in septic patients, unstimulated NET release from neutrophils was decreased by $46.3 \%(4.3 \% \pm 1.8$ SD vs. $8.2 \% \pm 2.9, p \leq 0.0001)$ and $48.1 \%(4.9 \% \pm 2.5$ vs. $9.4 \% \pm 5.2, p=0.002)$ after 2 and $4 \mathrm{~h}$ compared to volunteers. mtDNA further decreased NET formation in neutrophils from septic patients ( $4.7 \% \pm 1.2$ to $2.8 \% \pm 0,8 ; p=$ 0.03), but did not alter NET formation in neutrophils from volunteers. Of note, using PMA, as positive control, we ensured that neutrophils were still able to form NETs, with NET formation increasing to $73.2 \%( \pm 29.6)$ in septic patients and $91.7 \%$ $( \pm 7.1)$ in volunteers $(p=0.22)$. Additionally, we show that serum nuclease activity (range: $0-6)$ was decreased in septic patients by $39.6 \%$ ( $3 \pm 2$ vs $5 \pm 0$, median and ICR, $p=0.0001$ ) compared to volunteers.

Conclusions: Unstimulated NET formation and nuclease activity are decreased in septic patients. mtDNA can further reduce NET formation in sepsis. Thus, neutrophils from septic patients show decreased NET formation in vitro despite diminished nuclease activity in vivo.

Trial registration: DRKS00007694, german clinical trials database (DRKS). Retrospectively registered 06.02.2015.

Keywords: Neutrophil extracellular traps, Sepsis, Nuclease activity, Mitochondrial DNA

\section{Background}

Neutrophils play a key role in the response to infection [1-3]. In addition to phagocytosis and intracellular killing of pathogens, they have been shown to actively release extracellular netlike structures ("NET-osis") that consist of a nuclear DNA backbone, histones, and granular proteins, that can entrap, immobilize, and even kill gram-positive and gram-negative bacteria, fungi, and parasites [4-6].

\footnotetext{
* Correspondence: linda.cox@uk-essen.de

${ }^{1}$ Klinik für Anästhesiologie und Intensivmedizin, Universität Duisburg-Essen \& Universitätsklinikum Essen, Hufelandstraße 55, D-45122 Essen, Germany

Full list of author information is available at the end of the article
}

However, the regulation, if any, of the amount of circulating NETs is largely unknown. In mice with severe sepsis, depending on proinflammatory pathways NETs can be released [7], especially during the initial proinflammatory phase [8], and then degraded by serum nucleases $[4,8]$. The main function of human nucleases was said to be the destruction of extrinsic DNA, as ingested via the intestines. However, another important role of nucleases might be the decrease and thus the counterregulation of excessive NET concentration, i.e., to protect the body against negative effects of extracellular traps [8-10]. Accordingly, one might speculate that

(C) The Author(s). 2020 Open Access This article is distributed under the terms of the Creative Commons Attribution 4.0 International License (http://creativecommons.org/licenses/by/4.0/), which permits unrestricted use, distribution, and reproduction in any medium, provided you give appropriate credit to the original author(s) and the source, provide a link to the Creative Commons license, and indicate if changes were made. The Creative Commons Public Domain Dedication waiver (http://creativecommons.org/publicdomain/zero/1.0/) applies to the data made available in this article, unless otherwise stated. 
increased NET formation is associated with increased serum nuclease activity.

Some prior studies investigating NETs in human sepsis determined circulating free DNA (cfDNA) blood concentrations rather than actual NET formation itself $[10,11]$. This methodological approach is questionable as cfDNA also includes other non-NET-related types of human DNA, like genomic or mitochondrial DNA (mtDNA) [12, 13]. Thus, all types of circulating DNAs contributed to the measured amount of cfDNA in these studies, which then was found to be increased in septic patients $[10,11]$ or mice [8]. mtDNA, a damage-associated molecular pattern (DAMP), is increased in patients with severe trauma and activates neutrophils $[14,15]$. As mtDNA impacts on immune pathways, it even might influence NET formation. Additionally, phorbol 12-myristate 13-acetate (PMA) an artificial and maximal NET stimulator is widely used as positive control to ensure proper viability of neutrophils [4].

Accordingly, we tested the hypotheses that 1) NET formation from neutrophils of septic patients is increased compared to healthy volunteers, both without stimulation and following incubation with mtDNA or phorbol 12-myristate 13-acetate (PMA; positive control) and 2) that serum nuclease activity is increased as well.

\section{Material and methods}

\section{Patients' and volunteers' characteristics}

Following local ethics committee approval (no. 09-4154) and study registration (German clinical trials database, DRKS no. 00007694), we included 18 consecutive septic patients admitted to our intensive care unit (ICU), as well as 27 healthy volunteers, mostly hospital staff, to this prospective, observational trial. Septic patients were eligible if they fulfilled the criteria of sepsis according to the Surviving Sepsis Campaign Guidelines [16]. The SOFA Score of the patients with sepsis averaged $14 \pm 2$ (mean \pm standard deviation), which is accompanied by a calculated $89,7 \%$ mortality [17]. All patients needed mechanical ventilation and vasoactive support, and 17 of 18 patients needed more than $0,1 \mu \mathrm{g} / \mathrm{kg} / \mathrm{min}$ norepinephrine alone or in combination with dopamine. 9 of 18 patients received haemodialysis. 8 of 18 Patients with sepsis died in the first 30 days after blood sampling (i.e., a $44 \%$ mortality). Volunteers were eligible if they did not suffer from any acute or chronic disease, had no vaccination within 14 days prior to blood withdrawal, and were not taking chronic medications (except for oral contraception pills in women). To exclude that volunteers had an unrecognized infection, white blood count and Creactive protein concentrations were measured and found to be within the normal reference range. Sample size was calculated based on preliminary experiments using an a priori power analysis using G-Power software (G*Power 3.2; Düsseldorf, Germany). An a priori $\alpha$-error $\mathrm{p}$ of 0.05 and a given power of $1-\beta$ of 0.95 revealed a sample size of 18 individuals per group, based on the determined effect size from preliminary results. Patients' and volunteers' characteristics are presented in Table 1.

\section{Procedures and measurements}

Within $24 \mathrm{~h}$ after first diagnosing sepsis blood was withdrawn for in vitro experiments and measurements, neutrophils were isolated (see below), and NET-formation assays were performed immediately, both in septic patients $(n=18)$ and healthy volunteers $(n=27)$. For the measurement of serum nuclease activity blood was stored on ice upon withdrawal, centrifuged $(2000 \mathrm{~g}$ for $10 \mathrm{~min}$ ), and serum was frozen at $-80^{\circ} \mathrm{C}$ until analysis.

\section{Isolation of neutrophils}

Primary blood-derived neutrophils were isolated from fresh blood by density gradient centrifugation using Polymorphprep $^{\text {Tx }} \quad$ (Progen Biotechnik, Heidelberg, Germany), as described previously [18]. For in vitro NET formation assays, the neutrophils were seeded on polyL-lysine-coated glass slides in 24-well plates at a concentration of $5 \times 10^{5}$ cells per well $(250 \mu \mathrm{l})$ in RPMI 1640 medium (Thermo Fisher Scientific Inc., Waltham, MA) at $37^{\circ} \mathrm{C}$ and $5 \% \mathrm{CO}_{2}$, and NET formation was analyzed after incubation for 2 and $4 \mathrm{~h}$, respectively.

\section{Isolation of mitochondrial DNA}

To harvest a high amount of pure mtDNA, the cultivated human cell line HepG2 was used, as described previously [13]. The cultivation took place in RPMI medium mixed with $10 \%$ fetal calf serum (FKS) and $1 \mathrm{mM}$ sodium pyruvate (Thermo Fisher Scientific Inc., Waltham, MA) and cells were stored at $37^{\circ} \mathrm{C}$ and $5 \% \mathrm{CO}_{2}$. For the isolation of mitochondria, the Mitochondria Isolation Kit for Cultured Cells (Thermo Fisher Scientific Inc., Waltham, MA) was used according to product description. Purified mitochondria sediments were collected and stored at $4{ }^{\circ} \mathrm{C}$ for $24 \mathrm{~h}$ before mtDNA was isolated using the DNeasy Blood \& Tissue Kit (Qiagen, Hilden, Germany) following the manufacturer's protocol. The mtDNA concentration was determined photometrically at a wavelength of $280 \mathrm{~nm}$ (Biophotometer Plus, Eppendorf, Hamburg, Germany). mtDNA was stored at $-20^{\circ} \mathrm{C}$.

\section{Assessment and induction of NET formation}

NET formation was assessed in septic patients $(n=18)$, and healthy volunteers $(n=27)$ without stimulation (baseline), and following incubation with mtDNA (final concentration: $10 \mu \mathrm{g} /$ well), Furthermore, phorbol 12myristate 13-acetate (PMA, $25 \mathrm{nM}$ final concentration), a recognized NET formation inductor [19], was used as positive control, to ensure overall stimulability of neutrophils. In detail, $250 \mu \mathrm{l}$ RPMI medium including either 
Table 1 Infection related characteristics of patients and volunteers

\begin{tabular}{|c|c|c|c|}
\hline Variable & Healthy volunteers $(n=27)$ & Patients with sepsis $(n=18)$ & $p$-value \\
\hline Mean age, years & $33 \pm 14$ & $49 \pm 17$ & $<0.0001$ \\
\hline Females / males, N (\%) & $20(74) / 7(26)$ & $2(11) / 16(89)$ & $<0.0001$ \\
\hline Leukocyte blood concentration $\left(10^{3} \mu \mathrm{I}^{-1}\right)$ & $6 \pm 1$ & $18 \pm 15$ & 0.002 \\
\hline Neutrophil blood concentration $\left(10^{3} \mu^{\left.l^{-1}\right)}\right.$ & $3 \pm 0.5$ & $17 \pm 7$ & $\leq 0.0001$ \\
\hline Immature neutrophils (\% of all neutrophils) & $\mathrm{n} / \mathrm{a}$ & $10 \pm 12$ & \\
\hline C-reactive protein serum concentration $\left(\mathrm{mgl}^{-1}\right.$ ) & $\leq 0.5 \pm 0$ & $22 \pm 13$ & $\leq 0.0001$ \\
\hline Procalcitonin serum concentration (ng ml ${ }^{-1}$ ) & $\mathrm{n} / \mathrm{a}$ & $56 \pm 96$ & \\
\hline SAPS $\|$ & $\mathrm{n} / \mathrm{a}$ & $34 \pm 10$ & \\
\hline SOFA & $\mathrm{n} / \mathrm{a}$ & $14 \pm 2$ & \\
\hline 30-day survival (\%) & 100 & 56 & \\
\hline Gram-positive sepsis N (\%) & $\mathrm{n} / \mathrm{a}$ & $6(33)$ & \\
\hline Gram-negative sepsis N (\%) & $\mathrm{n} / \mathrm{a}$ & $5(28)$ & \\
\hline Mixed bacterial sepsis N (\%) & $\mathrm{n} / \mathrm{a}$ & $3(17)$ & \\
\hline Viral sepsis N (\%) & $\mathrm{n} / \mathrm{a}$ & 0 & \\
\hline Mycotic sepsis N (\%) & $\mathrm{n} / \mathrm{a}$ & $2(11)$ & \\
\hline Sepsis without microbial detection N (\%) & $\mathrm{n} / \mathrm{a}$ & $2(11)$ & \\
\hline $\begin{array}{l}\text { Source of infection } \\
\text { 1) Pneumonia, } N\end{array}$ & $\mathrm{n} / \mathrm{a}$ & 8 & \\
\hline $\begin{array}{l}\text { 2) Acute respiratory distress syndrome (ARDS) subsequent to } \\
\text { prior Pneumonia, but only microbial detection in blood, } N\end{array}$ & $\mathrm{n} / \mathrm{a}$ & 4 & \\
\hline 3) Urinary tract infection, $\mathrm{N}$ & $\mathrm{n} / \mathrm{a}$ & 1 & \\
\hline 4) Abdominal infection & $\mathrm{n} / \mathrm{a}$ & 2 & \\
\hline 5) Thoracic infection & $\mathrm{n} / \mathrm{a}$ & 1 & \\
\hline 6) No focus detected & $\mathrm{n} / \mathrm{a}$ & 2 & \\
\hline
\end{tabular}

Data from 27 healthy volunteers and 18 septic patients. Data are presented as numbers, percentages, or means \pm standard deviation.

$p$-values relate to Student's t-test for unpaired samples, where applicable.

the respective stimulating agent or vehicle (negative control) was added to $250 \mu \mathrm{l}$ of cell suspensions $\left(5 \times 10^{5}\right.$ cells/well) in 24 well plates.

To achieve adhesion of neutrophils to the glass-insert all plates were centrifuged at room temperature $\left(22^{\circ} \mathrm{C}\right)$ at $512 \mathrm{~g}$. Incubation time was 2 and $4 \mathrm{~h}$ at $37^{\circ} \mathrm{C}$ and $5 \%$ $\mathrm{CO}_{2}$, respectively. Neutrophils and neutrophil derived structures were fixed with $150 \mu \mathrm{l}$ of $16 \%$ paraformaldehyde and the plates were stored at $5{ }^{\circ} \mathrm{C}$ until immunostaining.

\section{Visualization and quantification of NET formation}

Since the major backbone of NETs is the DNA, different DNA-intercalating dyes, for example, 4',6-Diamidino-2Phenylindole (DAPI), propidium iodide, SYTOX Orange, or SYTOX Green, are widely used to visualize NETs (de Buhr et al., 2016). Importantly, we have recently shown that cationic antimicrobial peptides, for example, the cathelicidin LL-37, which are associated with NETs, block the binding of DNA-intercalating dyes to the NETs and thereby hamper their visualization (Neumann et al., 2014). Therefore, in the present study we here used an antibody-based technique with an antibody directed against histone-DNA complexes, as a typical marker for NETs. NET visualization and quantification was performed as described previously [18], In detail, cell preparations were washed three times with phosphate-buffered saline (PBS), and permeabilized by incubation with $2 \%$ bovine serum albumin (BSA) in $0.2 \%$ Triton X-100/PBS for $45 \mathrm{~min}$ at room temperature. A mouse monoclonal anti DNA/histone H1 complex (mouse IgG2a anti DNA/histone antibody, Merck Millipore, Darmstadt, Germany) was added and cells were incubated overnight at $4{ }^{\circ} \mathrm{C}$. After washing the cells three times with PBS an Alexa-Fluor-488-labelled goat-antimouse antibody (Thermo Fisher Scientific Inc., Waltham, MA) was added for $45 \mathrm{~min}$ at room temperature. Cells were then washed again and slides mounted in ProlongGold ${ }^{\bullet}$ antifade with DAPI (Invitrogen, Carlsbad, CA) and NET formation was analyzed using fluorescence microscopy (Leica TCS SP5 confocal microscope and Zeiss Anxioveit $200 \mathrm{M}$ non-confocal fluorescence microscope). From each slide three images were randomly selected. Using an antibody against histone-DNA complexes [20], different stages of NET-formation can 
be identified based on characteristic morphological changes of the nucleus upon stimulation and before release of NETs [18]. During the NET-formation process, disintegration of the nuclear membrane occurs concomitantly with cytoplasmic granule dissolution, allowing NET components to mix in the cytoplasm. The normal lobulated nuclear structure is then broken and a delobulated nuclear form can be found in those cells that are in the early stages of NET-formation. The criteria used for NET-positive cells were: Positively stained green nucleus plus a less dense nucleus (loss of lobulation) or a loss of the round shape of the nucleus plus an increased size of the nucleus, or an occurrence of a distinct extracellular off-shoot [21]. Using this method, the simple counting of dead necrotic cells is excluded based on 1) the antibody staining used and 2) the morphological characteristic of the cells. Data are presented as a percentage of cells showing NET formation related to all neutrophils of an image. For statistical analysis, the mean value of 6 images was used for calculation of average values for each condition and individual.

\section{Quantification of nuclease activity}

Serum nuclease activity of the consecutive septic patients and healthy volunteers was quantified by gel electrophoresis. As negative control tris-(hydroxymethyl)aminomethan (TRIS)-buffer (300 mM TRIS, $50 \mathrm{mM}$ calcium chloride, $50 \mathrm{mM}$ magnesium chloride) was used. A dilution series of the DNase I (Sigma Aldrich, St. Louis, $\mathrm{MO}$ ), with an activity range from 2 to 0,0035 units $/ \mathrm{ml}$, served as a positive control. TRIS-buffer and calf thymus DNA (Sigma Aldrich, St. Louis, MO) in a concentration of $1 \mathrm{mg} / \mathrm{ml}$ was added to the serum samples as well as to the positive and the negative controls. Samples were incubated for $18 \mathrm{~h}$ at $37^{\circ} \mathrm{C}$. A phenol chloroform (Carl Roth, Karlsruhe, Germany) extraction was used to separate the DNA from proteins. The resulting fluid phase was mixed with loading buffer (Thermo Fisher Scientific Inc., Waltham, MA) and added to an agarose gel pocket (1\%), followed by gel electrophoresis at $100 \mathrm{~V}$ for $30 \mathrm{~min}$.

For semiquantification of serum nuclease activity, we compared the gel lane from samples to a dilution series of DNase I. The possible activity range was related to categories 1-6. This grading correlates with DNase I activity of zero (activity range 1 ), $<0,007 \mathrm{U} / \mathrm{ml}$ (range 2), 0,007 U/ $\mathrm{ml}$ (range 3), 0,007-0,015 $\mathrm{U} / \mathrm{ml}$ (range 4), 0,015-0,06 U/ $\mathrm{ml}$ (range 5), and $\geq 0,06 \mathrm{U} / \mathrm{ml}$ (range 6), respectively.

\section{Statistical analysis}

Microsoft Excel 2016 (V16, Microsoft, Redmond, WA) and GraphPad Prism (V 6, GraphPad Software, San Diego, CA) were used for data analysis. Data are presented as means ( \pm standard deviation) unless indicated otherwise. The Student's two-tailed t-test for independent samples or, in case of violation of the normality assumption (as tested by the Kolmogorov-Smirnov and Shapiro-Wilk tests), the Wilcoxon signed rank test was used. Potential associations between serum nuclease activity and clinicopathogenic variables of septic patients like C-reactive protein and procalcitonin serum concentrations were determined using Spearman correlation analysis. Null hypotheses were rejected and statistical significance assumed with an a priori alpha error $\mathrm{p}$ of less than 0.05 .

\section{Results}

\section{Unstimulated NET formation}

Unstimulated NET formation from neutrophils of septic patients was significantly lower than in healthy volunteers (all $p<0.0001$ ). In detail, after $2 \mathrm{~h}$ NET formation in septic patients was decreased by $46.3 \%(4.3 \% \pm 1.8$ vs. $8.2 \% \pm 2.9, p<0.0001)$ and $48.1 \%(4.9 \% \pm 2.5$ vs. $9.4 \% \pm$ $5.2, p=0.002)$ after $4 \mathrm{~h}$, compared to healthy volunteers (Fig. 1).

\section{NET formation following mtDNA stimulation} mtDNA decreased NET formation in neutrophils from septic patients $(4.7 \% \pm 1.2$ to $2.8 \% \pm 0,8 ; p=0.03$, Fig. 2$)$, which was not seen in healthy volunteers $(12.6 \% \pm 5.5$ vs. $17.4 \% \pm 8.5, p=$ n.s.)

\section{NET formation following PMA stimulation}

Positive control PMA increased NET formation to greater than $70 \%$ both in septic patients and healthy volunteers, indicating that neutrophils were vital and capable for NET formation following maximum artificial stimulation. As expected, maximum NET formation did not differ between groups (healthy volunteers: $91.7 \% \pm$ 7.1 vs. septic patients: $73.2 \% \pm 29.6, p=0.22$, Fig. 3 ).

\section{Nuclease activity}

Nuclease activity in septic patients was markedly decreased by $39.6 \%$ ( $3 \pm 2$ vs $5 \pm 0$; median \pm interquartile range; $p=0.0001$, Fig. 4). Interestingly, C-reactive protein $(r=-0.904 ; p=0.035)$ and procalcitonin serum concentrations $(r=-0.918 ; p=0.028)$ showed an inverse correlation with nuclease activity whereas the Simplified Acute Physiology Score II (SAPS II) did not $(r=-0.839$; $p=0.08)$.

\section{Discussion}

In septic patients 1) unstimulated ex vivo NET formation is decreased, 2) mtDNA further decreases NET formation, and 3) serum nuclease activity is decreased in septic patients compared to healthy volunteers. Thus, our results show, that both NET formation and degradation are diminished in septic patients.

Surprisingly, unstimulated neutrophils from septic patients released less NETs compared to neutrophils from 


\section{Extracellular Trap (NET) formation by neutrophils from volunteers and septic patients}

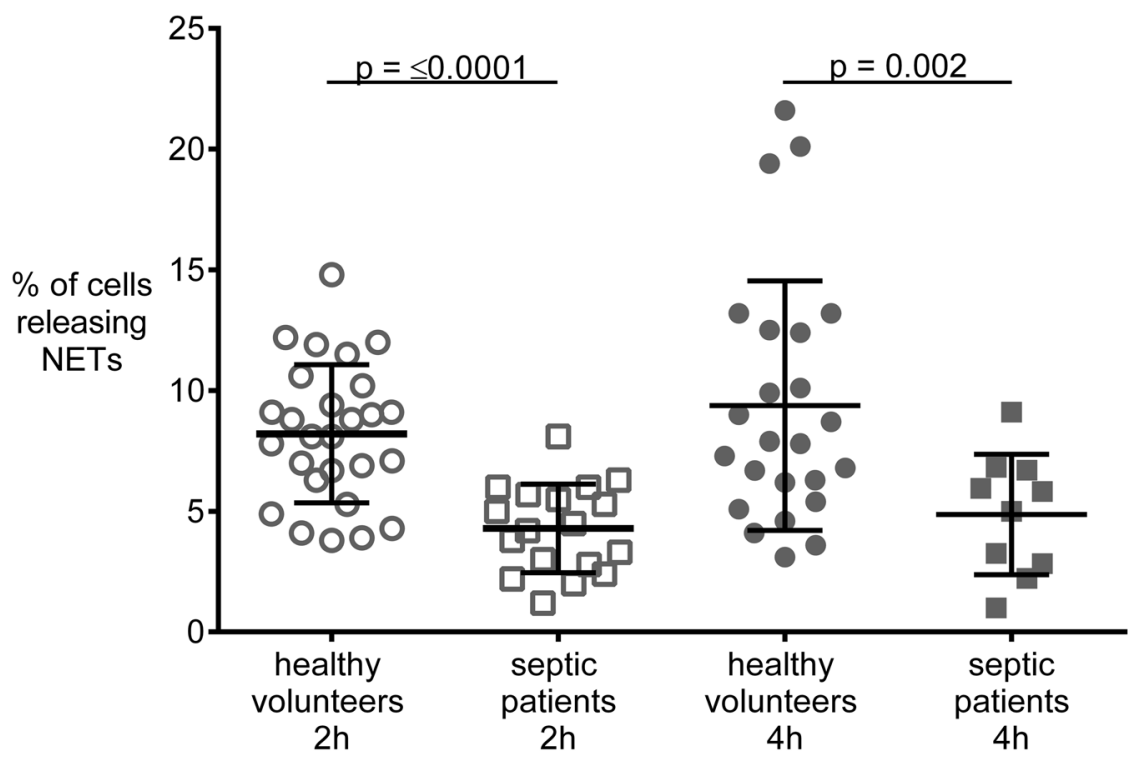

Fig. 1 Extracellular Trap (NET) formation by neutrophils obtained from blood of septic patients and volunteers following incubation for 2 and $4 \mathrm{~h}$. Neutrophils from septic patients released significantly less NETs than those from volunteers under baseline conditions both after incubation for 2 and $4 \mathrm{~h}$, respectively. Data are means $\pm \mathrm{SD}$

\section{Neutrophil Extracellular Trap (NET) formation following mtDNA stimulation in volunteers and septic patients}

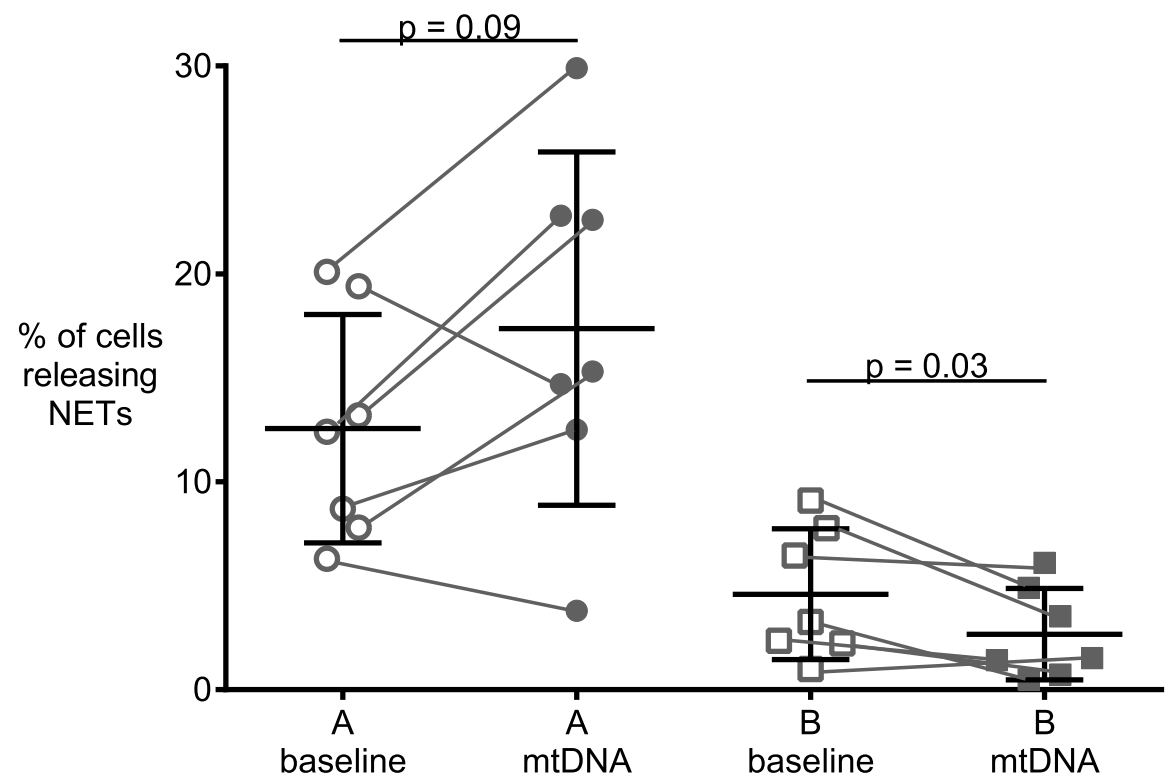

Fig. 2 Neutrophil Extracellular Trap (NET) formation in vitro at baseline (open symbols) and after $4 \mathrm{~h}$ of incubation with mitochondrial DNA ( $m$ tDNA, full symbols) in neutrophils from volunteers (left panel, A) and septic patients (right panel, B). While NET formation in neutrophils from volunteers $(A)$ is unaltered by mtDNA, mtDNA evoked a marginal decrease in NET formation in neutrophils from septic patients (B). Data are individual values and means \pm SD 


\section{Neutrophil Extracellular Trap (NET) formation evoked by PMA stimulation}

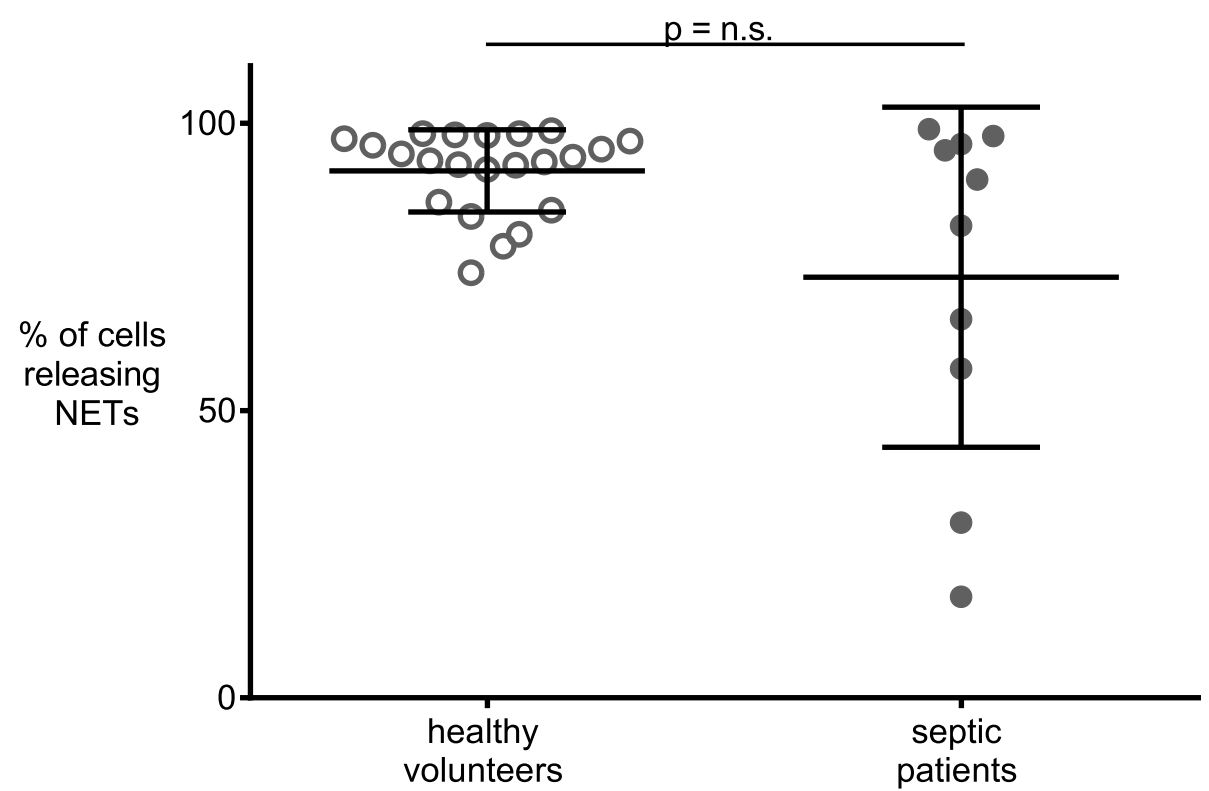

Fig. 3 Neutrophil Extracellular Trap (NET) formation following phorbol-myristate-acetate (PMA) stimulation. PMA (25 nM) led to a strong increase of NET formation, both in septic patients and volunteers, reaching NET formation in more than $70 \%$ of neutrophils. Thus, in spite of decreased NET formation under baseline conditions in septic patients, NET formation capacity in neutrophils from septic patients is still high and approaches that of PMA stimulated neutrophils from volunteers. Data are means \pm SD

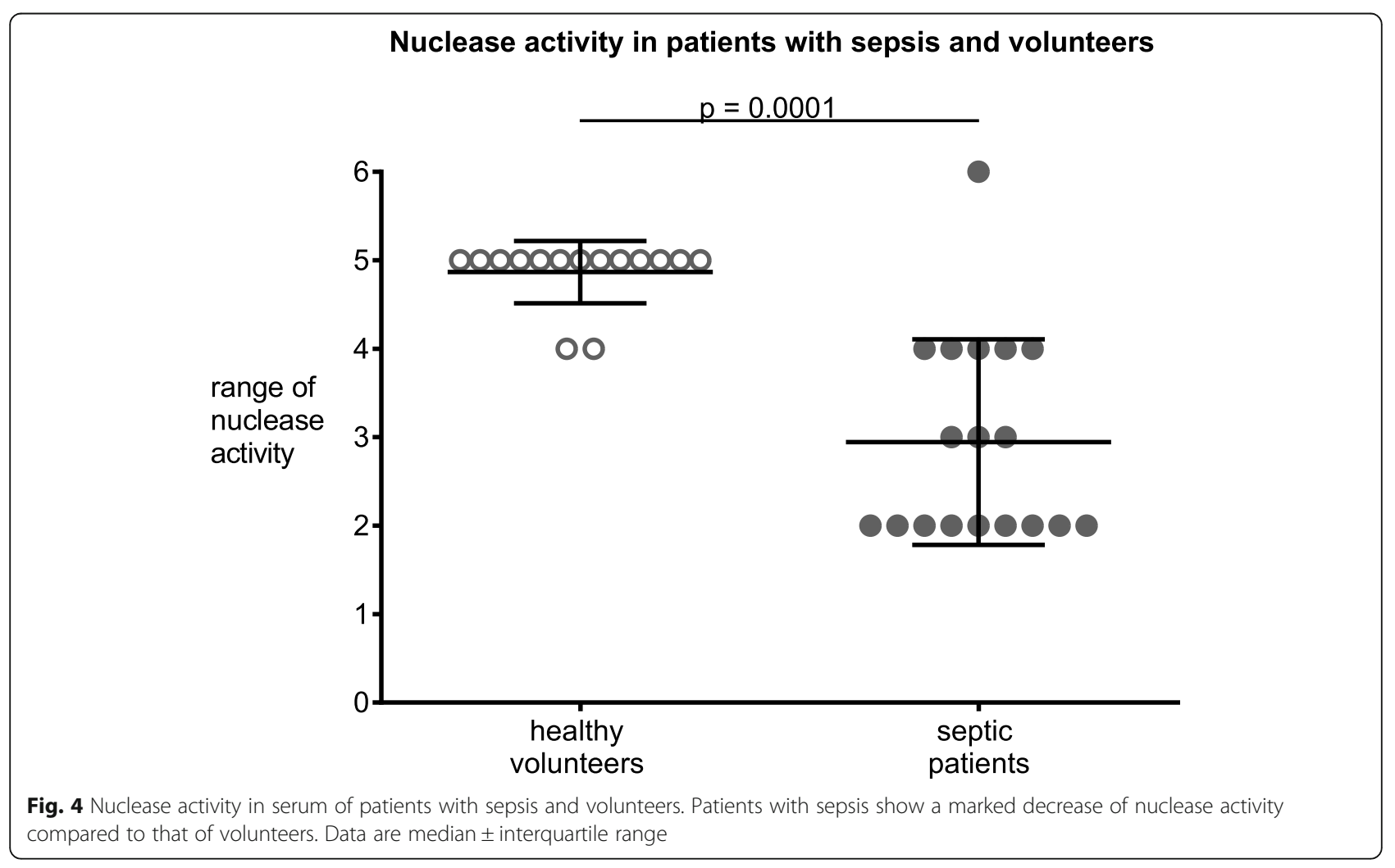


healthy volunteers. Therefore, we need to consider possible confounders. First, could serum proteins in septic patient's blood block NET formation. This is unlikely as we studied a standardized number of isolated and several times washed neutrophils. Thus, remaining serum is highly low and a depressant effect of serum components on ex vivo NET formation can almost be ruled out. Second, might a methodological aspect have led to decreased NETosis This is unlikely as well, as applying the extremely strong stimulant PMA, which is routinely used as positive control, cells performed maximal NETosis, which did not differ between groups. Thus, it is also unlikely, that the intracellular machinery for NET formation was dysfunctional in neutrophils from septic patients. By the same token, it is also unlikely that neutrophils isolated from septic patients were "exhausted" or still more "juvenile" than those from healthy volunteers since both groups had equally high PMA evoked NET formation.

Even more important we need to discuss our findings in context with recent publications. Some prior studies already focused on NET formation in sepsis [10, 11]. However, these studies did not use the gold standard of visualization and quantification of actual NETosis in vitro. In contrast, they used an easy approach and measured total circulating free DNA (cfDNA). As we and others have shown recently, in sepsis cfDNA includes other non-NET-related types of human DNA, like genomic or mitochondrial DNA (mtDNA) [13, 14]. Furthermore, cfDNA cannot discriminate between DNA motives actively released by neutrophils and increased concentrations i.e. related to cell death [22]. Thus, measuring the amount of total cfDNA does not equal NETosis. In the aforementioned publications, all types of circulating DNAs contribute to the measured amount of cfDNA, which than was found to be increased in septic patients $[10,11]$ or mice [8]. Another work showed increased concentrations of cfDNA in patients with sepsis despite decreased NET release, which supports our argumentation [23]. Thus, direct quantification of NETs in vivo should be used as methodological gold standard. In this respect, Gavillet et al. recently established a flow cytometric assay to directly visualize NETs [24]. This might be a feasible, less time consuming, and easier method for NET quantification in further studies.

Of note, Hashiba et al. measured both cfDNA and NET formation in septic patients by direct visualization using the approach of Fuchs as published previously [5]. Even, when the method is established, the specific PMA assay used did not induce maximal NETosis, both from neutrophils of septic and nonseptic individuals. This is in contrast to the definition of PMA as positive control for NET formation $[4,5,25]$. Thus, as published previously PMA evokes maximum NET release, as PMA is an extremely strong NET inductor and therefore maximum
NETosis is required following PMA stimulation to ensure cell viability prior to the experiment or adequate experimental setup $[4,5,25]$. Interestingly, results from NETosis and cfDNA measurement in this study differed, confirming the finding that cfDNA contains DNA from various sources.

In this regard mtDNA, a danger associated molecular pattern, is of particular importance, as mtDNA is known to have many effects on the immune system. It increases the TNF- $\alpha$, interleukin- $1 \beta$, and hypoxia-inducible factor$1 \alpha$ mRNA expression in humans [13]. It has also been shown that mtDNA strongly stimulates polymorphonuclear neutrophils [12]. Therefore, mtDNA, might stimulate NET formation. In contrast to our hypothesis, following mtDNA incubation, NET formation was decreased. However, basal, unstimulated, NET formation in our experiments was already low, so that we could only identify a modest drop in NETosis from neutrophils of septic patients. This is interesting, as Zhang et al. had shown that mitochondrial DAMPS, being a mixture of mtDNA and mitochondrial proteins stimulate neutrophils [12], whereas highly purified mtDNA alone, as we used it in our experiments, did not activate neutrophils [26]. As we and others have shown recently, mtDNA can induce immunosuppressive phenotypes, by inhibiting cytotoxic T-cell activity, both in wildtype mice and humans with sepsis $[12,13]$. Since mtDNA serum concentrations are increased in trauma and sepsis [13], and excessive NET formation might endanger the organism $[10,27]$, one might speculate that the body as a selfprotective feature does not form NETs in response to mtDNA.

Another explanation for reduced NET release in sepsis might be an acidotic $\mathrm{pH}$ of septic patients, as shown by Patel et al., who measured NET release ex vivo in a cohort of septic patients comparable to our cohort [23]. In fact, we included patients with septic shock, as all of them needed vasoactive medication. The SOFA score in our cohort was $14 \pm 2$ (mean \pm standard deviation) and we were able to show a comparable reduction of NET release. The septic patients we included also had an acidotic acid base status at the time of blood collection. Their $\mathrm{pH}$ was $7.3 \pm 0.09$ and the standardized base excess was $-3 \pm 5$ (means \pm standard deviation). No correlation was seen in our patients between the severity of acidosis and decreased NET release or 30-day mortality. Please note, however, that the assessment of the acid base status might be limited because 50\% (9 of 18) of our septic patients received continuous haemodialysis. Finally, we assessed serum nuclease activity, which we found to be decreased in septic patients. The combination of decreased basal NET formation and decreased serum nuclease activity is interesting at it might ensure that decreased basal NET formation is not further 
diminished by high nuclease serum concentrations. On first sight, our data appear to contrast those of Meng et al., reporting increased DNAse concentrations in septic mice [8]. However, in addition to potential differences between mice and human patients, it needs to be pointed out that we measured the nuclease activity and not the concentration irrespective of activity.

Finally, leukocyte and neutrophil counts differed between septic patients and healthy volunteers, with septic patients having increased blood neutrophil count $\left(17.2 \times 10^{3} / \mu \mathrm{l} \pm\right.$ 7.7 vs. $3.4 \times 10^{3} / \mu \mathrm{l} \pm 0.6 ; p<0.0001$ ). Thus, when calculating the product of basal NET release and neutrophil count both in septic patients and healthy volunteers, NET formation capacity would be higher in septic patients.

Thus, combination of decreased nuclease activity and increased neutrophil count might at least restore total in vivo NET formation capacity in septic patients despite decreased NET formation in vitro.

Our study has limitations. First, sepsis is a long lasting disease with an initial proinflammatory phase overlapping with later immunoparalysis [28-30]. Our analysis took place in the early proinflammatory phase, when blood neutrophil concentrations are usually increased [31]. Nevertheless, the decreased NETs release might possibly result from both a negative feedback mechanism as well as an immunosuppressive pattern. As stated above 30 -day mortality was $44 \%$, with the time of death following ICU admission ranging from day 1 to 30 . In detail, patients died on days $2,12,14,15,16,16,17$, and 29 . Thus, the time of death occurred at early, intermediate, and late time points. Accordingly, patients might have been in either immunostimulatory and immunosuppressive phases or in between. Furthermore, more and more evidence suggests that there is an early overlap of immunostimulatory and immunosuppressive patterns in sepsis. Hence, we do not know whether NET formation may be different during later phases of sepsis. In septic mice, cfDNA concentrations had decreased $48 \mathrm{~h}$ after induction of sepsis when compared to $24 \mathrm{~h}$, while DNAse concentration remained unaltered [8]. Obviously, while allowing in vitro measurements of NET formation in response to mediators in washed neutrophils and normalized to cell number, our experiments may not reflect NET behavior in the blood and tissues where different cytokine and cell interactions likely prevail. Accordingly, more studies are necessary to elucidate potential differences in NET formation in different microenvironments. Neutrophils of elderly human seem to release less NETs than those of young individuals [32]. In our cohort, we included more controls and these were not strictly age and gender matched to the patients with sepsis. While the age range of both groups overlapped healthy controls were younger. However, we feel that this is of limited importance as our participant's age ranges widely and one cannot allocate individuals to a "young" and "old" group. In fact, elderly human have been defined to be 65 years and older in other studies on NET release [33, 34].

In conclusion, neutrophils isolated from septic patients showed reduced NET formation at baseline compared to volunteers. mtDNA, an endogenous DAMP acting on TLR9 receptors, had a modest if any inhibitory effect on neutrophils from septic patients, and serum nuclease activity in septic patients was decreased. Decreased nuclease activity and increased neutrophil count, thus might be counterregulated by decreased basal NET formation. This may be important for protection of the organism from destructive effects of overwhelming NET release in sepsis.

\section{Abbreviations \\ BSA: Bovine serum albumin; cfDNA: Circulating free DNA; DAMP: Damage- associated molecular pattern; DAPI: 4',6-Diamidino-2-Phenylindole; FKS: Fetal calf serum; ICU: Intensive care unit; mtDNA: Mitochondrial DNA; NET: Neutrophil extracellular trap; PBS: Phosphate-buffered saline; PMA: Phorbol 12-myristate 13-acetate; TRIS-buffer: Tris-(hydroxymethyl)- aminomethan-buffer}

\section{Acknowledgements}

I would like to thank Ursula Brecklinghaus and Bärbel Hermann for their support in the laboratory work.

\section{Authors' contributions}

LEC carried out NETs quantification, isolation of mitochondrial DNA, statistical analysis and drafted the manuscript. KW carried out the gel electrophoresis for the quantification of nuclease activity and participated in the NETs quantification. LV and FR participated in NETs visualization and quantification. $A B$ and $A D$ participated in patient's selection and blood sampling. AE did laboratory supervision and participated in the design of the study. JP helped in coordination and drafting the manuscript. MvKB conceived of the study, and participated in its design and coordination and helped to draft the manuscript. STS also conceived of the study, and contributed in its design, coordination, statistical analysis and participated in drafting the manuscript. All authors read and approved the final manuscript.

\section{Funding}

Departmental sources, Klinik für Anästhesiologie \& Intensivmedizin, Universitätsklinikum Essen, Essen, Germany, with interest of research in sepsis associated processes, especially role NETs. We included patients of the intensive care unit and used the laboratories of the Klinik für Anästhesiologie \& Intensivmedizin. There was no influence of the funding body in design of the study, analysis, interpretation of data and in writing the manuscript.

\section{Availability of data and materials}

The final datasets supporting the conclusions of this article are included within the article and its additional files. The raw data that support the findings of this study are available from the corresponding author, LEC, upon reasonable request.

\section{Ethics approval}

No. 09-4154, the study was submitted to and approved by Ethik-Kommission, Medizinische Fakultät, Universität Duisburg-Essen. We obtained written informed consent from all participants.

Consent for publication

Not applicable.

Competing interests

The authors declare that they have no competing interests. 


\section{Author details}

"Klinik für Anästhesiologie und Intensivmedizin, Universität Duisburg-Essen \& Universitätsklinikum Essen, Hufelandstraße 55, D-45122 Essen, Germany. ${ }^{2}$ Institut für Physiologische Chemie, Stiftung Tierärztliche Hochschule Hannover, Bünteweg 2, D-30559 Hannover, Germany. ${ }^{3}$ Research Center for Emerging Infections and Zoonoses, Stiftung Tierärztliche Hochschule Hannover, Hannover, Germany. ${ }^{4}$ Klinik für Anaesthesiologie,

Ludwig-Maximilians-Universität München, Munich, Germany.

Received: 13 July 2019 Accepted: 9 December 2019

Published online: 13 January 2020

\section{References}

1. Smith JA. Neutrophils, host defense, and inflammation: a double-edged sword. J Leukoc Biol. 1994:56:672-86.

2. Nathan C. Neutrophils and immunity: challenges and opportunities. Nat Rev Immunol. 2006;6:173-82.

3. Schleimer RP, Freeland HS, Peters SP, Brown KE, Derse CP. An assessment of the effects of glucocorticoids on degranulation, chemotaxis, binding to vascular endothelium and formation of leukotriene B4 by purified human neutrophils. J Pharmacol Exp Ther. 1989;250:598-605.

4. Brinkmann V, Reichard U, Goosmann C, Fauler B, Uhlemann Y, Weiss DS, et al. Neutrophil extracellular traps kill bacteria. Science (New York, NY). 2004;303:1532-5.

5. Fuchs TA, Abed U, Goosmann C, Hurwitz R, Schulze I, Wahn V, et al. Novel cell death program leads to neutrophil extracellular traps. J Cell Biol. 2007; 176:231-41.

6. Urban CF, Reichard U, Brinkmann V, Zychlinsky A. Neutrophil extracellular traps capture and kill Candida albicans yeast and hyphal forms. Cell Microbiol. 2006:8:668-76.

7. Clark SR, Ma AC, Tavener SA, McDonald B, Goodarzi Z, Kelly MM, et al. Platelet TLR4 activates neutrophil extracellular traps to ensnare bacteria in septic blood. Nat Med. 2007;13:463-9.

8. Meng W, Paunel-Görgülü A, Flohé S, Hoffmann A, Witte I, Mackenzie C, et al. Depletion of neutrophil extracellular traps in vivo results in hypersusceptibility to polymicrobial sepsis in mice. Crit Care. 2012;16:R137.

9. Fuchs TA, Brill A, Wagner DD. Neutrophil extracellular trap (NET) impact on deep vein thrombosis. Arterioscler Thromb Vasc Biol. 2012;32:1777-83.

10. Czaikoski PG, Mota JM, Nascimento DC, Sonego F, Castanheira FV, Melo PH, et al. Neutrophil extracellular traps induce organ damage during experimental and clinical Sepsis. PLoS One. 2016;11:e0148142.

11. Hashiba M, Huq A, Tomino A, Hirakawa A, Hattori T, Miyabe H, et al. Neutrophil extracellular traps in patients with sepsis. J Surg Res. 2015;194: 248-54

12. Zhang $Q$, Raoof M, Chen Y, Sumi Y, Sursal T, Junger W, et al. Circulating mitochondrial DAMPs cause inflammatory responses to injury. Nature. 2010; 464:104-7.

13. Schäfer ST, Franken L, Adamzik M, Schumak B, Scherag A, Engler A, et al, Mitochondrial DNA: an endogenous trigger for immune paralysis. Anesthesiology. 2016;124:923-33.

14. Zhang Q, Itagaki K, Hauser CJ. Mitochondrial DNA is released by shock and activates neutrophils via p38 map kinase. Shock. 2010;34:55-9.

15. Nakahira K, Kyung SY, Rogers AJ, Gazourian L, Youn S, Massaro AF, et al. Circulating mitochondrial DNA in patients in the ICU as a marker of mortality: derivation and validation. PLoS Med. 2013;10:e1001577.

16. Dellinger RP, Levy MM, Rhodes A, Annane D, Gerlach H, Opal SM, et al. Surviving Sepsis campaign: international guidelines for management of severe sepsis and septic shock, 2012. Intensive Care Med. 2013;39:165-228.

17. Ferreira FL, Bota DP, Bross A, Melot C, Vincent JL. Serial evaluation of the SOFA score to predict outcome in critically ill patients. Jama. 2001;286: $1754-8$.

18. von Köckritz-Blickwede M, Chow O, Ghochani M, Nizet V. Visualization and functional evaluation of phagocyte extracellular traps. In: Kaufmann $\mathrm{S}$, Kabelitz D, editors. Immunology of Infection, vol. 3; 2010. p. 139-50.

19. Brinkmann V, Laube B, Abu Abed U, Goosmann C, Zychlinsky A. Neutrophil extracellular traps: how to generate and visualize them. JoVE. 2010. https:// doi.org/10.3791/1724.

20. Losman MJ, Fasy TM, Novick KE, Monestier M. Monoclonal autoantibodies to subnucleosomes from a MRL/Mp(-)+/+ mouse. Oligoclonality of the antibody response and recognition of a determinant composed of histones H2A, H2B, and DNA. J Immunol. 1992;148:1561-9.
21. Brogden G, Neumann A, Husein DM, Reuner F, Naim HY, von KockritzBlickwede M. Methods to study lipid alterations in neutrophils and the subsequent formation of neutrophil extracellular traps: Journal of Visualized Experiments : JoVE; 2017.

22. Hamaguchi S, Akeda Y, Yamamoto N, Seki M, Yamamoto K, Oishi K, et al. Origin of circulating free DNA in Sepsis: analysis of the CLP mouse model. Mediat Inflamm. 2015;2015:614518.

23. Patel JM, Sapey E, Parekh D, Scott A, Dosanjh D, Gao F, et al. Sepsis induces a Dysregulated neutrophil phenotype that is associated with increased mortality. Mediat Inflamm. 2018;2018:4065362.

24. Gavillet M, Martinod K, Renella R, Harris C, Shapiro NI, Wagner DD, et al. Flow cytometric assay for direct quantification of neutrophil extracellular traps in blood samples. Am J Hematol. 2015;90:1155-8.

25. Vollger L, Akong-Moore K, Cox L, Goldmann O, Wang Y, Schafer ST, et al. Iron-chelating agent desferrioxamine stimulates formation of neutrophil extracellular traps (NETs) in human blood-derived neutrophils. Biosci Rep. 2016;36. https://doi.org/10.1042/BSR20160031.

26. Prikhodko AS, Shabanov AK, Zinovkina LA, Popova EN, Aznauryan MA, Lanina NO, et al. Pure mitochondrial DNA does not activate human neutrophils in vitro. Biochemistry Biokhimiia. 2015;80:629-35.

27. Fuchs TA, Brill A, Duerschmied D, Schatzberg D, Monestier M, Myers DD, et al. Extracellular DNA traps promote thrombosis. Proc Natl Acad Sci U S A. 2010;107:15880-5

28. Boomer JS, To K, Chang KC, Takasu O, Osborne DF, Walton AH, et al. Immunosuppression in patients who die of sepsis and multiple organ failure. Jama. 2011:306:2594-605.

29. Boomer JS, Shuherk-Shaffer J, Hotchkiss RS, Green JM. A prospective analysis of lymphocyte phenotype and function over the course of acute sepsis. Crit Care. 2012;16:R112.

30. Ward PA. Immunosuppression in sepsis. Jama. 2011;306:2618-9.

31. Phillipson $M$, Kubes $P$. The neutrophil in vascular inflammation. Nat Med. 2011;17:1381-90.

32. Ortmann W, Kolaczkowska E. Age is the work of art? Impact of neutrophil and organism age on neutrophil extracellular trap formation. Cell Tissue Res. 2018:371:473-88.

33. Hazeldine J, Harris P, Chapple IL, Grant M, Greenwood H, Livesey A, et al. Impaired neutrophil extracellular trap formation: a novel defect in the innate immune system of aged individuals. Aging Cell. 2014;13:690-8.

34. Itagaki K, Kaczmarek E, Lee YT, Tang IT, Isal B, Adibnia Y, et al. Mitochondrial DNA released by trauma induces neutrophil extracellular traps. PLoS One. 2015;10:e0120549.

\section{Publisher's Note}

Springer Nature remains neutral with regard to jurisdictional claims in published maps and institutional affiliations.

Ready to submit your research? Choose BMC and benefit from:

- fast, convenient online submission

- thorough peer review by experienced researchers in your field

- rapid publication on acceptance

- support for research data, including large and complex data types

- gold Open Access which fosters wider collaboration and increased citations

- maximum visibility for your research: over $100 \mathrm{M}$ website views per year

At $\mathrm{BMC}$, research is always in progress.

Learn more biomedcentral.com/submissions 